\title{
1 Nuclear stabilisation of p53 requires a functional nucleolar
}

\section{2 surveillance pathway}

3

4 Katherine M. Hannan ${ }^{1,2,3}$, Priscilla Soo ${ }^{1}$, Mei S. Wong ${ }^{1,2,4 \S}$, Justine K. Lee ${ }^{5}$, Nadine Hein ${ }^{1}$, Maurits

$5 \quad$ Evers $^{1}$, Kira D. Wysoke ${ }^{1}$, Tobias D. Williams ${ }^{1 \S}$, Christian Montellese ${ }^{6}$, Lorey K. Smith ${ }^{2}$, Sheren J.

6 Al-Obaidi ${ }^{1}$, Lorena Núñez-Villacís ${ }^{1}$, Perlita Poh $^{1}$, Megan $\mathrm{Pavy}^{1}$, Jin-Shu He ${ }^{7}$, Kate M. Parsons ${ }^{7}$,

7 Jeannine Diesch ${ }^{2 \S}$, Gaetan Burgio ${ }^{8}$, Rita Ferreira ${ }^{1}$, Zhi-Ping Feng ${ }^{8}$, Cathryn M. Gould ${ }^{9}$, Piyush B.

8 Madhamshettiwar $^{9}$, Johan Flygare ${ }^{10}$, Thomas J. Gonda ${ }^{11 \S}$, Kaylene J. Simpson ${ }^{4,9}$, Ulrike Kutay ${ }^{6}$,

9 Richard B. Pearson ${ }^{1-4,12}$, Christoph Engel ${ }^{13}$, Nicholas J. Watkins ${ }^{5}$, Ross D. Hannan ${ }^{1-4,6,12,14^{* \#}}$ and

10 Amee J. George $e^{1,7,15 \#}$

11

12 Affiliations: ${ }^{1}$ ACRF Department of Cancer Biology and Therapeutics, John Curtin School of

13 Medical Research, Australian National University, Acton 2601 Australia; ${ }^{2}$ Oncogenic Signalling

14 and Growth Control Program, Peter MacCallum Cancer Centre, Melbourne 3000, Australia;

$15{ }^{3}$ Department of Biochemistry and Molecular Biology, University of Melbourne, Parkville 3010

16 Australia; ${ }^{4}$ Sir Peter MacCallum Department of Oncology, University of Melbourne, Parkville 3010

17 Australia; ${ }^{5}$ Institute for Cell and Molecular Biosciences, Newcastle University, Newcastle-Upon-

18 Tyne, UK; ${ }^{6}$ Department of Biology, Institute of Biochemistry, ETH Zurich, Zurich, Switzerland;

$19{ }^{7}$ ANU Centre for Therapeutic Discovery, John Curtin School of Medical Research, Australian

20 National University, Acton 2601 Australia; ${ }^{8}$ The John Curtin School of Medical Research,

21 Australian National University, Acton 2601 Australia; ${ }^{9}$ Victorian Centre for Functional Genomics,

22 Peter MacCallum Cancer Centre, Melbourne 3000, Australia; ${ }^{10}$ Lund Stem Cell Center, Lund

23 University, BMC A12, Lund, Sweden; ${ }^{11}$ School of Pharmacy, University of Queensland, Brisbane

244102 Australia; ${ }^{12}$ Department of Biochemistry and Molecular Biology, Monash University,

25 Clayton, 3800, Australia; ${ }^{13}$ Regensburg Center for Biochemistry, University of Regensburg, 93053 
26 Regensburg, Germany; ${ }^{14}$ School of Biomedical Sciences, University of Queensland, St Lucia 4067,

27 Australia; ${ }^{15}$ Department of Clinical Pathology, University of Melbourne, Parkville 3010 Australia.

$29{ }^{\S}$ Denotes current address: Mei S. Wong: Faculty of Medicine, SEGi University, 47810 Petaling

30 Jaya, Malaysia; Tobias D. Williams: Oncogenic Signalling and Growth Control Program, Peter

31 MacCallum Cancer Centre, Melbourne 3000, Australia, Jeannine Diesch: Cancer and Leukaemia

32 Epigenetics and Biology Program, Josep Carrerras Leukaemia Research Institute (IJC), Campus

33 ICO-GTP-UAB, Badalona, Spain, Thomas J. Gonda: University of South Australia Cancer

34 Research Institute, Adelaide 5000 Australia. *Denotes corresponding author; ${ }^{*}$ denotes equal

35 authorship.

37 Corresponding Author:

38 Professor Ross D. Hannan

39 ACRF Department of Cancer Biology and Therapeutics

40 The John Curtin School of Medical Research

41 Australian National University

42 Acton, 2601, Australia.

43 Email: ross.hannan@anu.edu.au

44

45 Keywords: Nucleolar surveillance pathway, nucleolus, p53, ribosome biogenesis, high-throughput 46 screening; ribosomal proteins, stress 


\section{Abstract}

48 The nucleolar surveillance pathway (NSP) monitors nucleolar fidelity and responds to nucleolar

49 stresses (i.e., inactivation of ribosome biogenesis) by mediating the inhibitory binding of ribosomal

50 proteins (RPs) to mouse double minute 2 homolog (MDM2), a nuclear-localised E3 ubiquitin

51 ligase, which results in p53 accumulation. Inappropriate activation of the NSP has been implicated

52 in the pathogenesis of collection of human diseases termed "ribosomopathies", while drugs that

53 selectively activate the NSP are now in trials for cancer. Despite the clinical significance, the

54 precise molecular mechanism(s) regulating the NSP remain poorly understood. Using genome-wide

55 loss of function screens, we demonstrate the ribosome biogenesis (RiBi) axis as the most potent

56 class of genes whose disruption stabilises p53. Furthermore, we identified a novel suite of genes

57 critical for the NSP, including a novel mammalian protein implicated in 5S ribonucleoprotein

58 particle (5S-RNP) biogenesis, HEATR3. By selectively disabling the NSP, we unexpectedly

59 demonstrate that a functional NSP is required for the ability of all nuclear acting stresses tested,

60 including DNA damage, to robustly induce p53 accumulation. Together, our data demonstrates that

61 the NSP has evolved as the dominant central integrator of stresses that regulate nuclear p53

62 abundance, thus ensuring RiBi is hardwired to cellular proliferative capacity. 
64

\section{Main}

Mutations in the potent tumour suppressor protein p53 and its effector pathways occur in the majority of human cancers, and are therefore the subject of intense investigation. A key mechanism by which p53 is regulated is at the level of protein stabilisation, through the MDM2 protein, which induces ubiquitination, and subsequently proteasomal degradation of p53. DNA damage from ionising radiation or certain chemotherapeutic agents lead to the amino-terminal phosphorylation of p53, which prevents MDM2 binding, and results in p53 stabilisation. This triggers a number of antiproliferative programs by activating or repressing key effector genes in a context-dependent manner ${ }^{1}$. The $\mathrm{p} 53-\mathrm{MDM} 2$ interaction is also antagonised by the tumour suppressor $\mathrm{p} 14^{\mathrm{ARF}}$ in response to oncogenic challenges ${ }^{2}$. More recently, a third mechanism of p53 stabilisation has been identified; the NSP, which is activated by acute disruptions to RiBi, resulting in inhibitory binding of certain RPs to MDM2, thus leading to increased abundance of nuclear p53 protein $^{3-5}$. In contrast to the former, the precise mechanisms underlying p53 stabilisation in response to the NSP are poorly understood. For example, the ribosomal proteins RPL5 and RPL11 have been implicated as the central regulators of the NSP through their participation in the 5S-RNP complex that binds to and inactivates MDM2 in response to nucleolar stress ${ }^{4,5}$. However, other RP and non-RP genes have also been implicated in regulating the NSP signalling process, suggesting the definitive mechanism is yet to be resolved. It is also unclear why loss or inactivation of only certain ribosomeassociated genes give rise to increased p53 stabilisation or are connected with ribosomopathies. Finally, the functional relationship of the NSP to the mechanisms underlying p53 stabilisation observed in response to 'classic' non-nucleolar stress pathways, such as proteasomal stress, hypoxia or DNA damage, is not clear.

To address these questions, we first identified the entire repertoire of genes whose deletion activates stress pathways leading to stabilisation of p53 in A549 (human lung adenocarcinoma, p53 wildtype) cells, by undertaking a high-throughput genome-wide RNA interference (RNAi) imaging- 
based screen measuring nuclear p53 accumulation using immunofluorescence ('p53 stabilisation screen', Fig. 1a, Supplementary File 1). The screen 'cut-off' was functionally defined as the minimum amount of p53 accumulation required to induce a significant cell-cycle defect (Supplementary Fig. 1a-d), which we identified as $\sim 2$-fold increase in p53 protein expression. Applying this cut-off $\left(\log _{2} \geq 1\right)$ to the screening dataset, 827 genes fulfilled this criterion (defined as 'p53 positive', Fig. 1b). We further interrogated the 'p53 positive' candidates to identify which molecular pathways/functions were enriched in the dataset using the KEGG network enrichment analysis feature of STRING ${ }^{6}$ (Fig. 1c, annotated version in Supplementary Fig. 2a \&

Supplementary File 2). This revealed an enrichment of six major classes of genes including: ribosome, nucleolus, proteasome, RNA splicing, cell cycle and RNA Polymerase II (Pol II). These classes were also broadly confirmed by gene ontology (GO) analysis (Fig. 1d, Supplementary File 3) and gene set enrichment analysis (GSEA, Supplementary Fig. 2b), resulting in GOs relating to RNP complex and RiBi, ribosomal RNA (rRNA) processing and rRNA metabolic processes being amongst the most significantly enriched. Moreover, intersecting our 'p53 positive' candidate list with the LOCATE subcellular localisation database ${ }^{7}$, we identified a significant over-representation of proteins localised to the nucleolus, nucleus and centrosome, and conversely, an underrepresentation of proteins located within the plasma membrane (Fig. 1e, Supplementary File 4). Collectively, these observations strongly support the notion that perturbations in $\mathrm{RiBi}$ and/or the nucleolus are a major, if not the most potent regulators of p53 accumulation.

We initially focussed specifically on RP genes given their prominence in the dataset; $\sim 80 \%$ of the RPs screened were 'p53 positive' when depleted, including RP genes associated with DiamondBlackfan Anaemia (DBA; e.g. RPS19, RPL35A, RPS7, RPS10, RPS24, RPS26, RPL26) ${ }^{8}$ (Fig. 2a). In a complementary approach, we evaluated the RPs using a quantitative total p53 assay (Alphascreen) to verify p53 expression, and observed a significant correlation between the results from both techniques (Fig. 2b, Supplementary Fig. 3a). In total, 77.3\% of the RPs specific to the 
$11660 \mathrm{~S}$ and $81.3 \%$ to the $40 \mathrm{~S}$ ribosomal subunits, when depleted, induced a 'p53 positive' phenotype,

117 implying that RPs to either subunit contributed similarly to the NSP p53 response. This finding is in

118 contrast to a study reporting that the large subunit RPs have a more profound p53 response when

119 depleted $^{9}$, though an arbitrary 5-fold increase in p53 was implemented as a 'cut off' in that study,

120 compared to our minimum physiologically relevant 2-fold cut off which was experimentally

121 determined. Importantly, the differential ability of the RPs when depleted to elicit p53 stabilisation

122 was not due to the inability of the siRNA to deplete the RP mRNA and protein (Fig. 2c,

\section{Supplementary Fig. 3b).}

124

125

126

128

129

130

131

132

133

134

135

136

137

138

139

140

141

We further examined whether the ability of a RP to induce the NSP correlated with the degree to which its depletion affected ribosome subunit biogenesis and function. We measured the abundance of the $40 \mathrm{~S}$ and $60 \mathrm{~S}$ ribosomal subunits, and the levels of mature ribosomes (80S) bound to mRNAs in polysomes following RP depletion. Consistent with the prediction, depletion of RPL21, RPS18 and RPS19, all of which induced robust stabilisation of p53, also robustly reduced the abundance of the corresponding $60 \mathrm{~S} / 40 \mathrm{~S}$ subunit in which they are located, as well as the number of polysomes

(Fig. 2d \& e). In contrast, depletion of RPL22 and RPL28, which failed to induce p53 stabilisation, did not impact on 60S biogenesis, nor the number of polysomes compared to siNT (Fig. $2 \mathbf{d} \& \mathbf{e}$ ). Exceptions to this were RPL5 and RPL11, whose knockdown failed to stabilise p53, even though 60S biogenesis was ablated. This observation is consistent with studies implicating 'free' RPL5 and RPL11 (i.e., not incorporated into a 60S) as essential for the NSP due to their ability to bind MDM2 as part of the $5 \mathrm{~S}-\mathrm{RNP}^{4,10-12}$.

We considered whether the location of a RP within the ribosome may predict their ability to disrupt ribosome assembly, and thus mediate p53 accumulation, upon depletion. To do this, we mapped the p53 intensity resulting from the knockdown of each RP onto the structure of the 60S and 40S subunits $^{13}$ (Fig. 2f). While RPS18 and RPS19 (corresponding to two of the highest p53 intensities 
142 observed in the screen) co-located in the same region within the $40 \mathrm{~S}$ subunit, there was no other

143 clear evidence supporting that the specific location of a RP in the ribosome would increase p53

144 stabilisation if depleted. Finally, we tested the hypothesis that RPs which integrate early into their

145 respective ribosomal subunit (i.e., within the nucleolus) might be essential for the core structure,

146 thus when depleted, would have the most profound effect on ribosome assembly and the NSP. By

147 comparing p53 intensity and the published timing of integration of each RP into the ribosome ${ }^{14}$

148 (Supplementary File 5), we demonstrated that the p53 levels were significantly higher following

149 knockdown of those RPs which integrate into their respective subunits during early nucleolar stages

150 of ribosome assembly (Fig. 2g). Thus, the ability of RPs to stabilise p53 correlated with their

151 propensity to cause significant disruption to ribosome subunit assembly when depleted. This may

152 explain, at least in part, why not all components of the ribosome, when mutated or deleted,

153 contribute to ribosomopathies. For example, RPL22 and RPL28, which do not perturb ribosome

154 subunit assembly when depleted, have not been associated with DBA to date.

156 Having identified the major classes of genes, including RPs, whose deletion leads to stabilisation of

$157 \mathrm{p} 53$, we next determined the role of the NSP in this process; a priori, we predicted that only those

158 genes directly involved in RiBi would be dependent on the NSP to stabilise p53 when depleted. To

159 address this question, we took an unbiased approach to identify the key components of NSP that can

160 be targeted to inactivate NSP-mediated p53 stabilisation. Accordingly, we performed a genome-

161 wide RNAi screen to determine the genes whose depletion suppressed p53 accumulation in

162 response to nucleolar stress induced by knockdown of RPS19, the prototypical DBA gene known to

163 induce NSP when depleted ${ }^{15-17}$ (termed 'modifiers of ribosomal stress' screen; Fig. 3a \&

164 Supplementary File 6). Using a cut-off for normalised p53 intensity of up to and including 0.5

$165\left(\log _{2}=-1\right.$, calculated based on 3 standard deviations (SD) above the positive control,

166 siTP53+siRPS19), we identified 64 genes essential for a functional NSP (Fig. 3a \&

167 Supplementary Fig. 4a). We rescreened these 64 candidates (outlined in Methods), to identify 
168

169

170

171

172

174

175

176

177

178

179

180

181

182

183

184

185

186

187

candidates which recapitulated the primary screen phenotype (i.e. suppressed p53 response when co-depleted with siRPS19) with two or more siRNA duplexes. Critically, in addition to TP53, both

RPL5 and RPL11 were the top ranked candidates which, when depleted, reduced p53 accumulation in response to NSP activation, while no other RPs reached this cut-off. This observation is in contrast to previous reports suggesting a variety of RPs regulate p53 stability (e.g. RPL23 ${ }^{18,19}$, $\mathrm{RPL}_{2} 6^{20,21}, \mathrm{RPS}^{22}{ }^{22} \mathrm{RPS}^{23}, \mathrm{RPS}^{24}{ }^{24}, \mathrm{RPS}^{25^{25}}, \mathrm{RPS}^{27 A^{26}}{ }^{\text {, RPS} 27 \text { and RPS27L }}{ }^{27}, \mathrm{RPS} 15, \mathrm{RPS} 20$ and RPL $37^{28}$ ). Our study, therefore, functionally defines RPL5 and RPL11 as the only RPs essential for the NSP, consistent with their proposed role in the 5S-RNP interaction with MDM2. Similarly, non-RP factors previously reported to be linked to RiBi and p53 activity (e.g. SRSF1, GLTSCR2 (PICT1), HEXIM1, MYBBP1A, RRP8 (NML) and NPM1 ${ }^{29-34}$ ) were not identified as high-ranking candidates, suggesting, at least in this system under the kinetics used for the assays, they are not essential for NSP-induced stabilisation of p53 and/or may play tissue or developmentally-specific roles in the NSP.

In addition to TP53, RPL5 and RPL11, we further validated a selection of candidates from the screen including HEATR3, RXRA and CIRH1A as bone fide modulators of the p53 response (Fig. 3b, Supplementary Fig. 4a, c-h \& i-n). HEATR3 (HEAT-repeat containing 3) was of significant interest as a novel direct regulator of the NSP and the 5S-RNP-MDM2 axis, as bioinformatic domain alignment suggested that HEATR3 is a human homolog of yeast symportin 1 (Syo1) protein, which enables import of rpL5 and rpL11 into the nucleus of Saccharomyces cerevisiae ${ }^{35,36}$, and acts as a scaffold for 5S-RNP biogenesis prior to incorporation into the pre-60S ribosomal subunit $^{35}$. To analyse any structural similarities between the human and yeast proteins, we modelled the HEATR3 structure based on the Chaetomium thermophilium Syo1 (ctSyo1) crystal structure ${ }^{36}$ using 'Modeller' (Fig. 4a \& Supplementary Fig. 5), which indicates the potential for RPL5 and RPL11 binding on opposite sides of the HEAT repeats similar to that shown for $\operatorname{ctSyo} 1^{35,36}$. In support of this model, co-immunoprecipitation experiments from A549 cells co-transfected with 

RPL1 1 and RPL5 bind to HEATR3 in situ (Fig. 4b). Moreover, depletion of HEATR3 partially phenocopied RPL5 and RPL11 knockdown, resulting in a marked reduction in 60S subunit production (Fig. 4c), the number of polysomes (Fig. 4d) and 5S-RNP binding to MDM2 (Fig. 4e \& f). The reduced efficacy of HEATR3 depletion to disrupt RiBi and NSP compared to RPL5 and RPL11 suggests there may also be HEATR3-independent pathways by which RPL5 and RPL11 can assemble into 5S-RNP. Even so, in toto, these findings strongly suggest that HEATR3 is a functional homolog of Syo1 and important for 60S assembly and NSP in human cells through its ability to interact with the 5S-RNP (Fig. $\mathbf{4 g}$ ).

Having functionally defined RPL5, RPL11 and HEATR3 as direct regulators of the NSP, we next used their depletion (RNAi) to determine how important a functional NSP is for stabilisation of p53 by stresses not traditionally implicated in RiBi. To do this, a representative selection of the 827 genes identified as 'p53 positive' (i.e., whose depletion increased p53 levels; Fig. 1b\&c; 232 genes representing nucleolar, ribosome, splicing, Pol II, proteasome, cell cycle and other gene classes) were rescreened to determine if their ability to stabilise p53 when depleted was dependent on the NSP (Fig. 5 \& Supplementary File 7). As expected, the ability of RP and other nucleolar/RiBirelated genes to robustly activate p53 when depleted was blocked when the NSP was inactivated by

215 Supplementary File 7). Thus, HEATR3 is necessary for 5S-RNP-MDM2 complex assembly in response to the disruption of many (but not all) RiBi proteins, consistent with the observations 
Critically, and unexpectedly, the ability of major classes of genes not traditionally associated with

221 the ribosome or the nucleolus (e.g., RNA splicing, cell cycle and Pol II, Fig.1c) to stabilise p53

222 following their depletion was also ablated upon co-knockdown of RPL5 or RPL11, and to a lesser

223 degree HEATR3 (Fig. 5a \& b, Supplementary File 7). The p53 suppression was not simply due to

224 reduced ribosome assembly (and therefore reduced p53 mRNA translation) as a consequence of

225 RPL5 or RPL11 depletion (Fig. 4c \& d), because co-depletion of RPS19 failed to blunt p53

226 accumulation, despite RPS19 depletion causing a similar defect in ribosomal subunit assembly and

227 polysomes (Fig. 2d \& e). Together, these data suggest the NSP is required for robust stabilisation of

228 p53 in response to the dysregulation of a large number of eukaryotic genes and cellular processes

229 that are not traditionally associated with RiBi.

231 Given these unexpected findings, we extended these studies to determine the requirement of a

232 functional NSP to mediate stabilisation of p53 in response to a broad range of pharmacological

233 agents and pathophysiologic stressors, including inhibitors of Pol I \& II, nucleic acid synthesis

234 inhibitors, agents that induce DNA damage, nuclear export inhibitors and proteotoxic stress.

235 Intriguingly, inactivation of the NSP by either RPL5 or RPL11 depletion ablated the ability of Pol

$236 \mathrm{I} / \mathrm{II}$ inhibitors, nuclei acid synthesis inhibitors and all classes of DNA damage-inducing agents to

237 stabilise $\mathrm{p} 53$. In contrast, the ability of proteotoxic stresses including proteasomal inhibitors,

238 nuclear transport inhibitors and heat shock to increase p53 levels were only moderately, or not at all

239 blunted by inactivation of the NSP (Fig. 5c \& Supplementary Fig. 6b). We also confirmed these

240 findings using a high-content screening-based approach (Supplementary Fig. 6c), where HEATR3

241 depletion also blunted the response, however, not as efficiently as RPL5/L11 depletion. We noted

242 that knockdown of RPL5 was consistently more potent at blocking the NSP compared to RPL11 or

243 HEATR3, suggesting RPL5 may modulate p53 by mechanisms in addition to inhibitory binding of

244 5S-RNP to MDM2. Consistent with this, we observed that knockdown of RPL5 but not RPL11 
245 significantly reduced p53 mRNA levels (Supplementary Fig. 6d), although the mechanism of this

246 reduction was not investigated further.

248 To further validate our results in a model of NSP inactivation (other than RPL5 and RPL11

249 depletion), we used embryonic fibroblasts (MEFs) isolated from mice harbouring the Mdm2 ${ }^{\mathrm{C} 305 \mathrm{~F}}$

250 mutation, which disrupts RPL5 and RPL11 (ergo 5S-RNP) binding to MDM2, thereby inactivating

251 the $\mathrm{NSP}^{4,12}$. We again tested a complement of nuclear and physiological stressors (Fig. 5d \&

252 Supplementary Fig. 6e) in these cells. Consistent with RPL5/RPL11 knockdown, the Mdm2 ${ }^{\mathrm{C} 305 \mathrm{~F}}$

253 mutation prevented the p53 response upon exposure to Pol I/II inhibitors, nucleic acid synthesis

254 inhibitors and all classes of DNA damage inducing agents, but not proteotoxic stress. Taken

255 together, the data indicates that an intact NSP is required for the stabilisation of p53 in response to a

256 broad range of cellular stresses, not just ribosomal/nucleolar stress. Notably, the quantitative effect

257 of $\mathrm{Mdm} 2^{\mathrm{C} 305 \mathrm{~F}}$ mutation to blunt p53 accumulation in response to stress more closely reflected the

258 effect of RPL11 depletion than RPL5 depletion, consistent with the conclusions above that RPL5

259 may modulate p53 by mechanisms in addition to inhibitory binding of 5S-RNP to MDM2.

261 In summary, using global screening approaches, we have identified the complement of genes and 262 pathways functionally required for stabilisation of p53 in response to the canonical NSP. Our data 263 definitively demonstrate that RPL5 and RPL11 do not induce p53 stabilisation when depleted, and 264 are the only RPs essential for functional NSP to stabilise $\mathrm{p} 53^{4,12,37}$. Furthermore, we demonstrate 265 that one of the top hits, HEATR3, is a potential ribosome assembly factor required for mammalian 266 60S ribosomal subunit assembly through binding of RPL5 and RPL11. Consistent with an essential 267 role for HEATR3 in NSP-mediated stabilisation of p53, HEATR3 depletion leads to reduced 268 association of the 5S-RNP with MDM2. 
270 Critically, by inactivating the NSP, we demonstrate that pharmacological agents and

271 pathophysiological conditions leading to genotoxic stress, as well as the majority of genes whose

272 loss-of-function stabilises p53, do so in an NSP-dependent fashion. Our data provides experimental

273 support to Rubbi and Milner's original hypothesis that the nucleolus, through the NSP, is a

274 universal stress sensor responsible for $\mathrm{p} 53$ homeostasis within cells ${ }^{38}$. Thus, we conclude that the

275 well-described mechanisms of genotoxic stress which induce extensive post-translational regulation

276 of $\mathrm{p} 53$, thereby modulating its interaction with MDM2, are insufficient in the absence of a

277 functioning NSP to robustly stabilise p53. The exception to this rule appears to be pathological

278 conditions and pharmacologic agents that result in proteasomal stress, which stabilise p53 largely

279 independently of the NSP. This is consistent with MDM2-mediated degradation of p53 being

280 dependent on a functioning proteasome to degrade ubiquitinated p53.

282 The differential ability of ribosome components to induce p53 stabilisation following their

283 depletion correlated directly with the degree of disruption of RiBi and ribosome assembly. By

284 extrapolation, we propose that all nuclear acting- pathological conditions, -pharmacologic agents or

285 genetic inactivating lesions stabilise p53 in a 5S-RNP-MDM2 dependent fashion, through

286 disruption of RiBi. Consistent with this, ribosomal DNA (rDNA) is highly sensitive to DNA

287 damage (a single lesion in the rDNA is sufficient to cause cell cycle arrest ${ }^{39}$ ), and most cytotoxic

288 drugs and pathologic conditions that induce DNA damage have been reported to cause defects in

289 RiBi. We propose that the nucleolus functions as the cellular equivalent of a sentinel or "canary in

290 the coal mine" to detect a broad range of cellular stresses and mediate stabilisation of p53. In this

291 model, the nucleolus acts a sensitivity gauge, whereby stresses such as DNA damage can only

292 stabilise p53 if the stress is of sufficient magnitude to perturb RiBi/nucleolar function, thereby

293 preventing minor cellular insults from inappropriately inhibiting proliferation. Given that $\mathrm{RiBi}$ is

294 the most energy-expensive process a cell undertakes, the evolution of such a mechanism also

295 ensures RiBi remains hardwired to proliferative capacity through p53 activity. 
297 Finally, due to the central role RiBi and the NSP plays in the regulation of p53, we suggest a

298 paradigm-shift in thinking is required for how this axis contributes to cancer pathogenesis. Due to

299 the pervasive stress tumour cells are exposed to, we propose that overcoming NSP-induced p53

300 activation is likely to be a very frequent step in malignant transformation. Indeed, RP genes are

301 hemizygously deleted in $43 \%$ of human cancers, and almost always in concert with TP53

302 mutations, while such RP deletions are infrequent in TP53-intact tumours ${ }^{40,41}$. This is consistent

303 with chronic activation of the NSP in response to RP deletion being incompatible with malignant

304 transformation and negatively selected for unless p53 is inactivated. 


\section{Acknowledgements}

Thanks to the Captain Courageous Foundation (captaincourageous.com.au) in particular, Jessica and Jeff Bond, and Bill Steele for their ongoing support and funding for this project. We would like to acknowledge the founding and current members of the Australian Diamond Blackfan Anaemia (ADBA) program, consisting of Sheren J. Al-Obaidi, Luen Bik To, Sarah C.E. Bray, Richard J.

Mdm2 mouse strain used in this work. We would also like to thank George Thomas (IDIBELL,

Spain) for his valuable discussions.

We would like to thank the Imaging and Cytometry Facility (Dr Harpreet Vohra and Mr Michael

Devoy), the ANU Bioinformatics Consultancy (Dr Zhi-Ping Feng and Mr Cameron Jack), and the staff at the Australian Phenomics Facility, the ACRF Biomolecular Resource Facility and the ANU

Centre for Therapeutic Discovery at the John Curtin School of Medical Research at the Australian

322 National University for their assistance. We would also like to thank the ACRF Victorian Centre for

323 Functional Genomics (Ms Jennii Luu and Mr Daniel Thomas), the Molecular Genomics Core (Dr

324 Gisela Mir Arneau and Ms Aga Borcz), the Bioinformatics Core (Mr Jason Ellul) and the Advanced

325 Centre for Cancer Cell Isolation and Flow Cytometry (Ms Viki Milovac and Ms Sophie Curcio)

326 located at the Peter MacCallum Cancer Centre for their technical assistance, and all members of the

327 Engel lab, especially Philipp Becker and Mona Höcherl.

329 This work was supported by funding from the Captain Courageous Foundation and the National 330 Health and Medical Research Council (NHMRC) of Australia (Project Grants \#1100654, \#1158732, 
332 R.B.P (\#1058586). C.E. is supported by the 'Emmy-Noether-Programme', DFG grant no. EN

333 1204/1-1. U.K. was supported by the Swiss National Science Foundation (SNSF) (grant

334 31003A_166565 and the NCCR 'RNA and disease'). N.J.W is supported by funding from the

335 DBAF, DBA UK and the BBSRC (BB/R00143X/1). A.J.G is supported by a Captain Courageous

336 Foundation Fellowship.

338 The Victorian Centre for Functional Genomics (K.J.S.) is funded by the Australian Cancer

339 Research Foundation (ACRF) and Phenomics Australia, through funding from the Australian

340 Government's National Collaborative Research Infrastructure Strategy (NCRIS) program and the

341 Peter MacCallum Cancer Centre Foundation. The ANU Centre for Therapeutic Discovery (A.J.G)

342 is funded by the ACRF, Australian National University and ACT Health.

\section{Author Contributions}

K.M.H, R.D.H and A.J.G conceived and designed the study and wrote the manuscript; all other authors have reviewed and edited the manuscript. K.J.S, R.D.H and A.J.G. designed the high-

348 throughput screening approaches utilised in this study. A.J.G, P.S, J.H and K.M.P. performed the

349 high-throughput screens/high-content imaging assays and developed the image analysis pipelines.

350 M.E., L.K.S, Z-P.F, C.M.G, P.B.M, K.J.S and A.J.G performed the analysis and visualisation of

351 screening data. M.S.W. and A.J.G designed and executed the validation of screening candidates and

352 HEATR3 co-immunoprecipitation analysis. J.K.L and N.J.W. performed the co-

353 immunoprecipitation analysis of 5S rRNA with MDM2. C.M., U.K and A.J.G performed the

354 ribosome shading and assembly analyses. C.E. performed the HEATR3/Syo1 sequence alignments

355 and predictive modelling. K.M.H., P.S, M.S.W, T.D.H, and P.P. performed the ribosomal subunit

356 and polysomal analyses. P.S, M.S.W, N.H, K.D.W, T.D.W, S.J.A, L.N.V, P.P, M.P, G.B, R.F and 
357 A.J.G performed the molecular biology and biochemical experiments. J.F, T.J.G., K.J.S, U.K.,

358 R.B.P., N.J.W., R.D.H. and A.J.G. contributed valuable interpretation and academic discussion.

359 R.D.H. and A.J.G. co-supervised the project.

360

361

\section{Disclosure Statement}

363

364 R.D.H is a Chief Scientific Advisor of Pimera, Inc. (San Diego, CA). All other authors have no 365 disclosures to report.

366 


\section{References}

368

3691 Zilfou, J. T. \& Lowe, S. W. Tumor suppressive functions of p53. Cold Spring Harb

$370 \quad$ Perspect Biol 1, a001883, doi:10.1101/cshperspect.a001883 (2009).

3712 Dai, M. S. et al. Physical and functional interaction between ribosomal protein L11 and the

372 tumor suppressor ARF. J Biol Chem 287, 17120-17129, doi:10.1074/jbc.M111.311902

373 (2012).

3743 Boulon, S., Westman, B. J., Hutten, S., Boisvert, F. M. \& Lamond, A. I. The nucleolus under stress. Mol Cell 40, 216-227, doi:10.1016/j.molcel.2010.09.024 (2010).

3764 Sloan, K. E., Bohnsack, M. T. \& Watkins, N. J. The 5S RNP couples p53 homeostasis to 377 ribosome biogenesis and nucleolar stress. Cell Rep 5, 237-247, 378 doi:10.1016/j.celrep.2013.08.049 (2013).

3795 Donati, G., Peddigari, S., Mercer, C. A. \& Thomas, G. 5S ribosomal RNA is an essential 380 component of a nascent ribosomal precursor complex that regulates the Hdm2-p53 381 checkpoint. Cell Rep 4, 87-98, doi:10.1016/j.celrep.2013.05.045 (2013).

3826 von Mering, C. et al. STRING: a database of predicted functional associations between 383 proteins. Nucleic Acids Res 31, 258-261, doi:10.1093/nar/gkg034 (2003).

3847 Sprenger, J. et al. LOCATE: a mammalian protein subcellular localization database. Nucleic 385 Acids Res 36, D230-233, doi:10.1093/nar/gkm950 (2008).

3868 Ulirsch, J. C. et al. The Genetic Landscape of Diamond-Blackfan Anemia. Am J Hum Genet 387 103, 930-947, doi:10.1016/j.ajhg.2018.10.027 (2018).

3889 Nicolas, E. et al. Involvement of human ribosomal proteins in nucleolar structure and p53389 dependent nucleolar stress. Nat Commun 7, 11390, doi:10.1038/ncomms11390 (2016).

39010 Bursac, S. et al. Mutual protection of ribosomal proteins L5 and L11 from degradation is 391 essential for p53 activation upon ribosomal biogenesis stress. Proc Natl Acad Sci U S A 109, 20467-20472, doi:10.1073/pnas.1218535109 (2012). 
39311 Fumagalli, S., Ivanenkov, V. V., Teng, T. \& Thomas, G. Suprainduction of p53 by

394 disruption of $40 \mathrm{~S}$ and $60 \mathrm{~S}$ ribosome biogenesis leads to the activation of a novel G2/M

395 checkpoint. Genes Dev 26, 1028-1040, doi:10.1101/gad.189951.112 (2012).

39612 Macias, E. et al. An ARF-independent c-MYC-activated tumor suppression pathway

397 mediated by ribosomal protein-Mdm2 Interaction. Cancer Cell 18, 231-243,

398 doi:10.1016/j.ccr.2010.08.007 (2010).

39913 Khatter, H., Myasnikov, A. G., Natchiar, S. K. \& Klaholz, B. P. Structure of the human 80S

$400 \quad$ ribosome. Nature 520, 640-645, doi:10.1038/nature14427 (2015).

40114 de la Cruz, J., Karbstein, K. \& Woolford, J. L., Jr. Functions of ribosomal proteins in

402 assembly of eukaryotic ribosomes in vivo. Annu Rev Biochem 84, 93-129,

403 doi:10.1146/annurev-biochem-060614-033917 (2015).

40415 Jaako, P. et al. Disruption of the 5S RNP-Mdm2 interaction significantly improves the 405 erythroid defect in a mouse model for Diamond-Blackfan anemia. Leukemia 29, 2221-2229, 406 doi:10.1038/leu.2015.128(2015).

40716 Jaako, P. et al. Mice with ribosomal protein S19 deficiency develop bone marrow failure 408 and symptoms like patients with Diamond-Blackfan anemia. Blood 118, 6087-6096, 409 doi:10.1182/blood-2011-08-371963 (2011).

41017 Sjogren, S. E. et al. Glucocorticoids improve erythroid progenitor maintenance and dampen 411 Trp53 response in a mouse model of Diamond-Blackfan anaemia. Br J Haematol 171, 517$412 \quad$ 529, doi:10.1111/bjh.13632 (2015).

41318 Dai, M. S. et al. Ribosomal protein L23 activates p53 by inhibiting MDM2 function in 414 response to ribosomal perturbation but not to translation inhibition. Mol Cell Biol 24, 7654415 7668, doi:10.1128/MCB.24.17.7654-7668.2004 (2004).

41619 Jin, A., Itahana, K., O'Keefe, K. \& Zhang, Y. Inhibition of HDM2 and activation of p53 by 417 ribosomal protein L23. Mol Cell Biol 24, 7669-7680, doi:10.1128/MCB.24.17.7669$418 \quad 7680.2004(2004)$. 
41920 Ofir-Rosenfeld, Y., Boggs, K., Michael, D., Kastan, M. B. \& Oren, M. Mdm2 regulates p53

m20 mRNA translation through inhibitory interactions with ribosomal protein L26. Mol Cell 32,

421 180-189, doi:10.1016/j.molcel.2008.08.031 (2008).

42221 Zhang, Y. et al. Negative regulation of HDM2 to attenuate p53 degradation by ribosomal

423 protein L26. Nucleic Acids Res 38, 6544-6554, doi:10.1093/nar/gkq536 (2010).

42422 Yadavilli, S. et al. Ribosomal protein S3: A multi-functional protein that interacts with both 425 p53 and MDM2 through its KH domain. DNA Repair (Amst) 8, 1215-1224, 426 doi:10.1016/j.dnarep.2009.07.003 (2009).

42723 Chen, D. et al. Ribosomal protein S7 as a novel modulator of p53-MDM2 interaction:

428 binding to MDM2, stabilization of $\mathrm{p} 53$ protein, and activation of p53 function. Oncogene

$429 \quad$ 26, 5029-5037, doi:10.1038/sj.onc.1210327 (2007).

43024 Zhou, X., Hao, Q., Liao, J., Zhang, Q. \& Lu, H. Ribosomal protein S14 unties the MDM2431 p53 loop upon ribosomal stress. Oncogene 32, 388-396, doi:10.1038/onc.2012.63 (2013).

43225 Zhang, X. et al. Identification of ribosomal protein S25 (RPS25)-MDM2-p53 regulatory 433 feedback loop. Oncogene 32, 2782-2791, doi:10.1038/onc.2012.289 (2013).

43426 Sun, X. X., DeVine, T., Challagundla, K. B. \& Dai, M. S. Interplay between ribosomal 435 protein $\mathrm{S} 27 \mathrm{a}$ and MDM2 protein in p53 activation in response to ribosomal stress. $J$ Biol 436 Chem 286, 22730-22741, doi:10.1074/jbc.M111.223651 (2011).

43727 Xiong, X., Zhao, Y., He, H. \& Sun, Y. Ribosomal protein S27-like and S27 interplay with 438 p53-MDM2 axis as a target, a substrate and a regulator. Oncogene 30, 1798-1811, 439 doi:10.1038/onc.2010.569 (2011).

44028 Daftuar, L., Zhu, Y., Jacq, X. \& Prives, C. Ribosomal proteins RPL37, RPS15 and RPS20 441 regulate the Mdm2-p53-MdmX network. PLoS One 8, e68667, 442 doi:10.1371/journal.pone.0068667 (2013). 
44329 Fregoso, O. I., Das, S., Akerman, M. \& Krainer, A. R. Splicing-factor oncoprotein SRSF1

444 stabilizes p53 via RPL5 and induces cellular senescence. Mol Cell 50, 56-66,

445 doi:10.1016/j.molcel.2013.02.001 (2013).

$44630 \quad$ Kumazawa, T. et al. Novel nucleolar pathway connecting intracellular energy status with 447 p53 activation. J Biol Chem 286, 20861-20869, doi:10.1074/jbc.M110.209916 (2011).

$44831 \quad$ Kuroda, T. et al. RNA content in the nucleolus alters p53 acetylation via MYBBP1A.

449 EMBO J 30, 1054-1066, doi:10.1038/emboj.2011.23 (2011).

45032 Lew, Q. J. et al. Identification of HEXIM1 as a positive regulator of p53. J Biol Chem 287, $451 \quad 36443-36454$, doi:10.1074/jbc.M112.374157 (2012).

45233 Sasaki, M. et al. Regulation of the MDM2-P53 pathway and tumor growth by PICT1 via 453 nucleolar RPL11. Nat Med 17, 944-951, doi:10.1038/nm.2392 (2011).

45434 Lindstrom, M. S. NPM1/B23: A Multifunctional Chaperone in Ribosome Biogenesis and 455 Chromatin Remodeling. Biochem Res Int 2011, 195209, doi:10.1155/2011/195209 (2011).

45635 Calvino, F. R. et al. Symportin 1 chaperones 5S RNP assembly during ribosome biogenesis 457 by occupying an essential rRNA-binding site. Nat Commun 6, 6510, 458 doi:10.1038/ncomms7510 (2015).

$45936 \quad$ Kressler, D. et al. Synchronizing nuclear import of ribosomal proteins with ribosome $460 \quad$ assembly. Science 338, 666-671, doi:10.1126/science.1226960 (2012).

46137 Teng, T., Mercer, C. A., Hexley, P., Thomas, G. \& Fumagalli, S. Loss of tumor suppressor 462 RPL5/RPL11 does not induce cell cycle arrest but impedes proliferation due to reduced 463 ribosome content and translation capacity. Mol Cell Biol 33, 4660-4671, 464 doi:10.1128/MCB.01174-13 (2013).

46538 Rubbi, C. P. \& Milner, J. Disruption of the nucleolus mediates stabilization of p53 in 466 response to DNA damage and other stresses. EMBO J 22, 6068-6077, 467 doi:10.1093/emboj/cdg579 (2003). 
46839 van Sluis, M. \& McStay, B. A localized nucleolar DNA damage response facilitates

469 recruitment of the homology-directed repair machinery independent of cell cycle stage.

G70 Genes Dev 29, 1151-1163, doi:10.1101/gad.260703.115 (2015).

47140 Ajore, R. et al. Deletion of ribosomal protein genes is a common vulnerability in human

472 cancer, especially in concert with TP53 mutations. EMBO Mol Med 9, 498-507,

473 doi:10.15252/emmm.201606660 (2017).

47441 Fancello, L., Kampen, K. R., Hofman, I. J., Verbeeck, J. \& De Keersmaecker, K. The

475 ribosomal protein gene RPL5 is a haploinsufficient tumor suppressor in multiple cancer

476 types. Oncotarget 8, 14462-14478, doi:10.18632/oncotarget.14895 (2017).

477 
bioRxiv preprint doi: https://doi.org/10.1101/2021.01.21.427535; this version posted January 21, 2021. The copyright holder for this preprint (which was not certified by peer review) is the author/funder. All rights reserved. No reuse allowed without permission.

\section{Hannan et al Fig. 1}

a

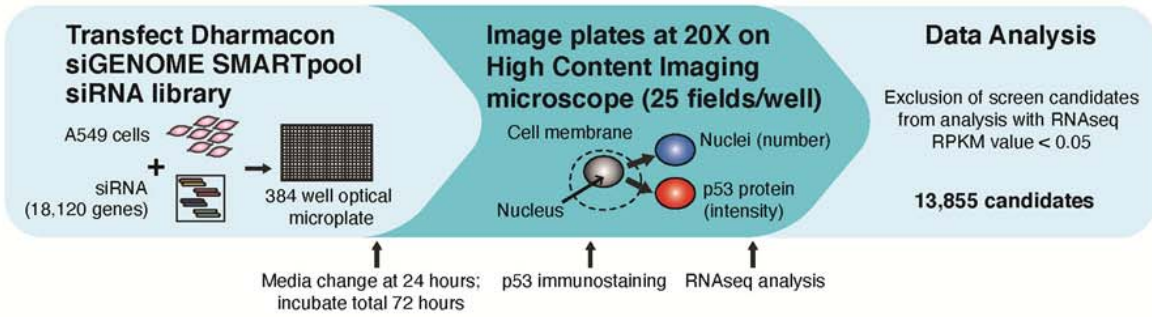

\section{b}

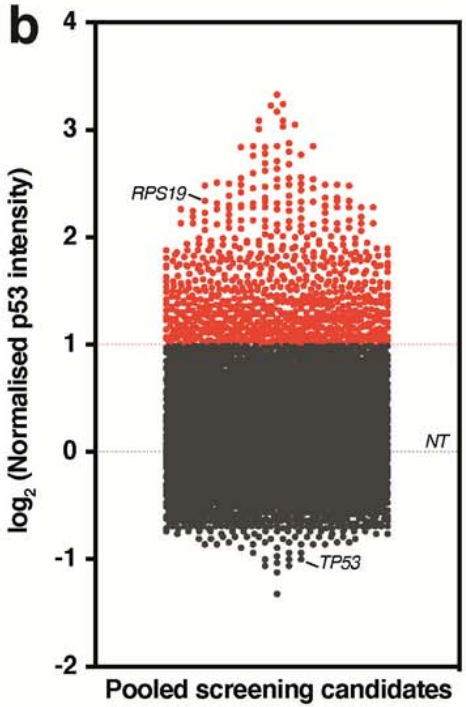

d

\section{GO Term}

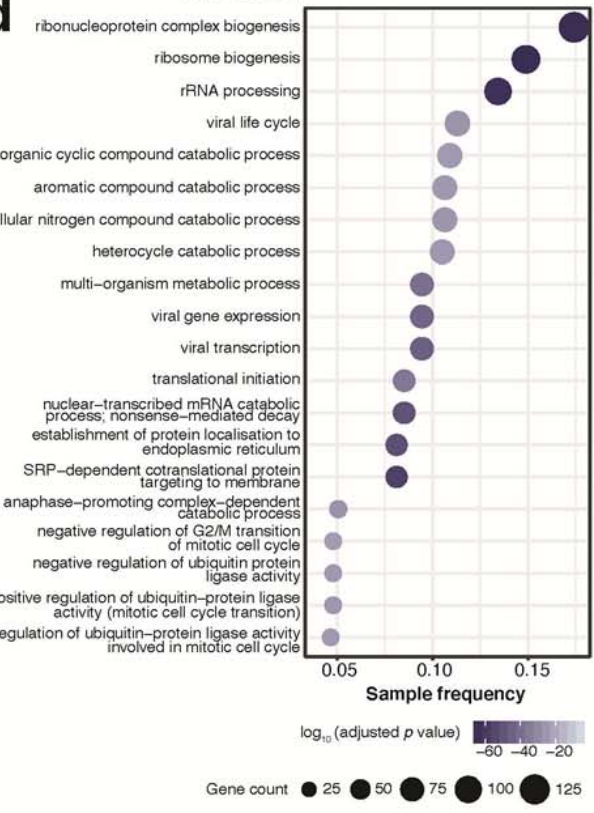

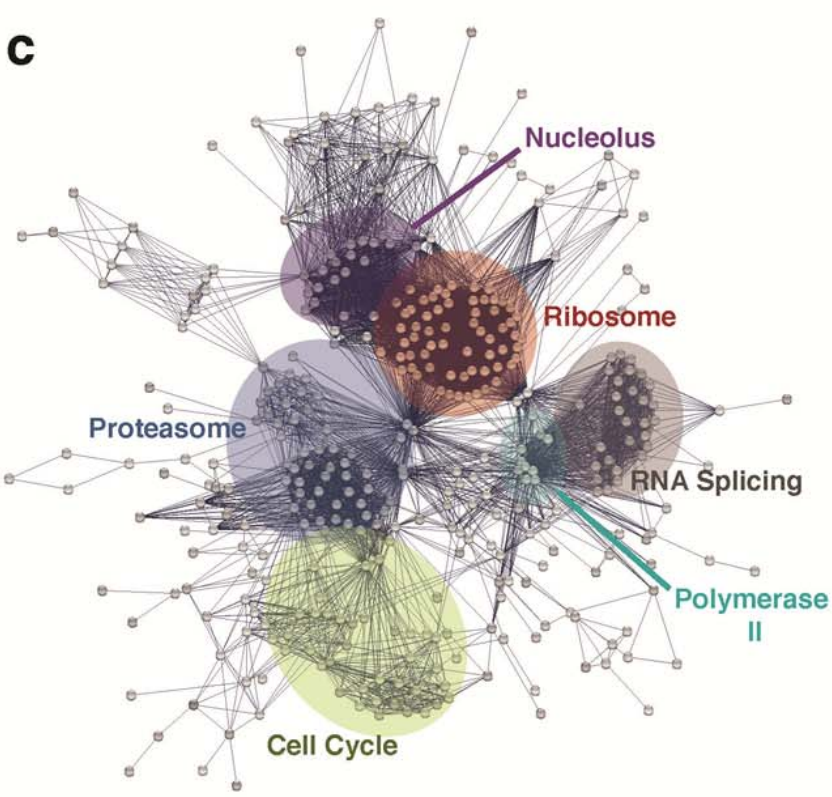

e
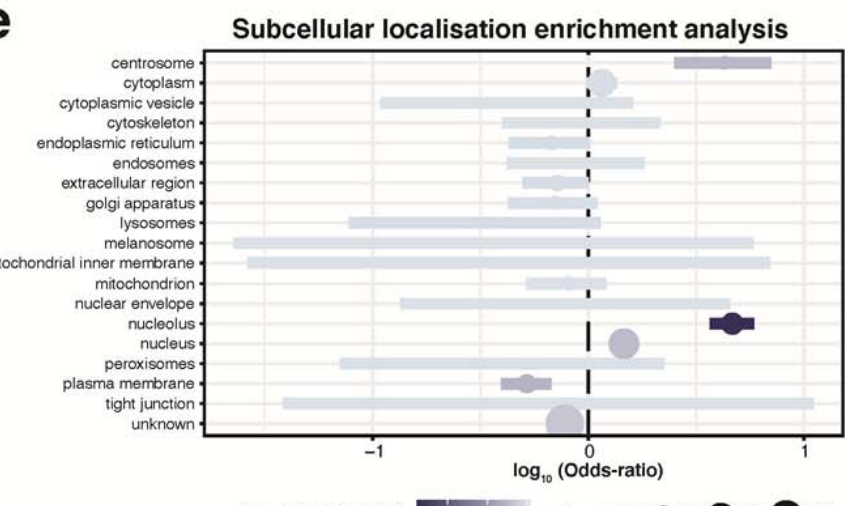

$\log _{10}$ (adjusted $\rho$ value) 
482 Figure 1: A genome-wide high-throughput screen reveals ribosome biogenesis and the 483 nucleolus as central components for modulating p53 stabilisation. Schematic of the genome484 wide high throughput screening approach in A549 (p53 wild-type) cells (a). A549 cells were 485 transfected with the genome-wide Dharmacon siGENOME SMARTpool siRNA library for 72 486 hours, then nuclear p53 intensity and cell number measured using an immunofluorescence-based 487 high-content (microscopy) imaging approach, with data normalised to non-targeting siRNA (NT) 488 transfected cells ('p53 stabilisation screen'). After intersection with RNAseq data from NT489 transfected cells (a cut-off of reads per kilobase per million, RPKM, of 0.05 or greater, to ensure 490 that candidates analysed are expressed in these cells), we determined the 'expressed' screening 491 candidates to be 13,855 . The 'expressed' candidates are graphed in (b); the top candidates (coloured 492 in red) are those which were 2-fold or greater $\left(\log _{2}=1\right) ; 827$ candidates in total. The top candidates 493 were then subjected to STRING (protein-protein interaction database) analysis using the KEGG 494 network enrichment analysis feature, and visualised in Cytoscape (described in Methods, c) to 495 identify clusters of similar proteins in the dataset (note: a fully annotated version of this figure is 496 located in Supplementary Figure 2A). Gene ontology (GO) analysis of the top candidates was then 497 performed, and simplified for graphical representation of the 'Biological Process' data using 498 ClusterProfiler (d, approach outlined in Methods), which depicts the gene ontologies versus the 499 gene ratio (ratio of number of query genes in the GO term and the total number of query genes). 500 Subcellular localisation enrichment analysis of the top candidates from the screen (using the 501 LOCATE database, e) was also performed; the $\log _{10}$ odds-ratio (OR) reflects the amount of 502 enrichment/depletion $(<0$ indicates under-representation, $>0$ indicates over-representation of query 503 genes in the corresponding category). The coloured bars represent $95 \%$ confidence intervals. 
Hannan et al Fig. 2

a

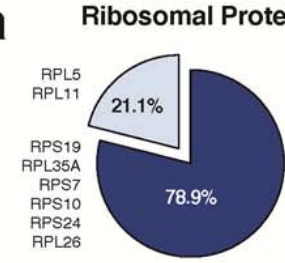

$\square$ p53 positive $\square$ p53 negative

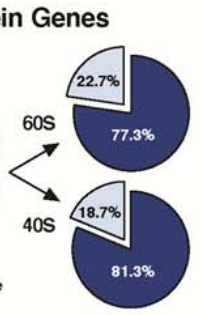

b

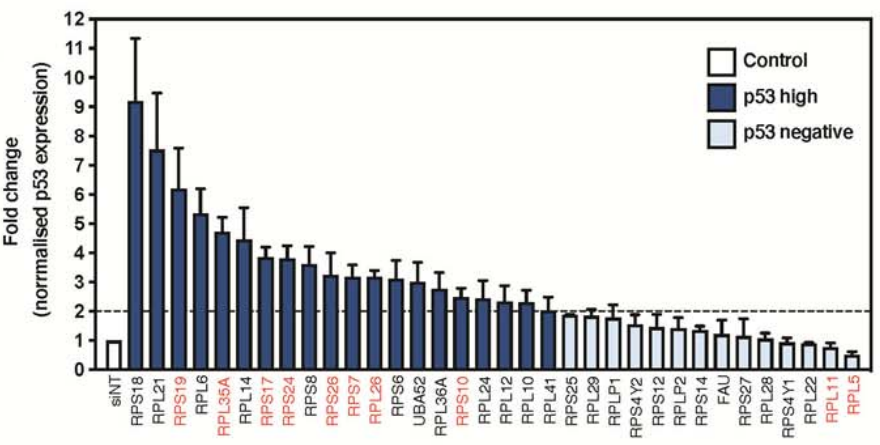

C
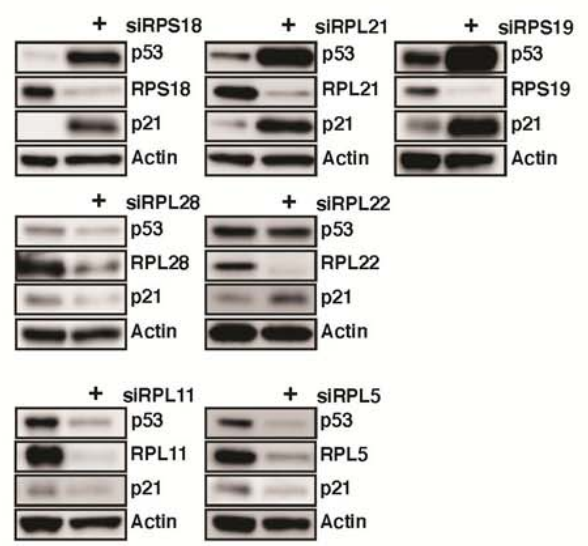

d

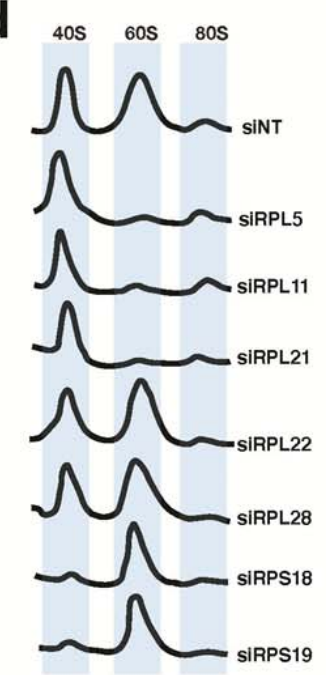

e

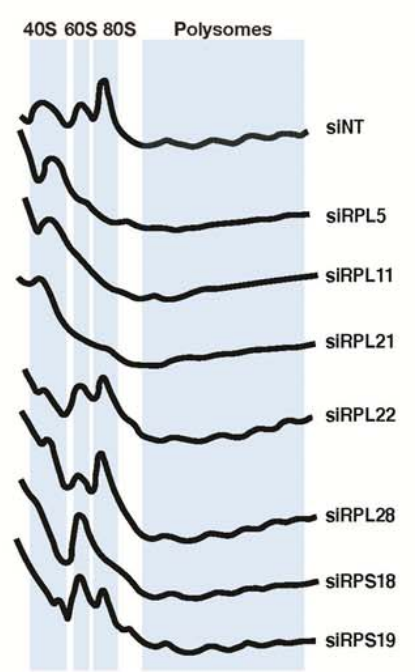

\section{$\mathbf{f}$}

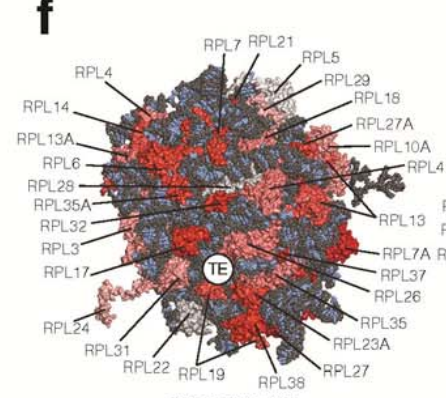

6OS SOLVENT

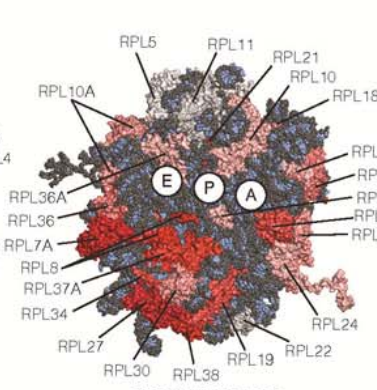

60 INTERFACE

\begin{tabular}{|c|}
\hline 1 \\
\hline $13 \quad 5 \quad 7 \quad 9$ \\
p53 intensity (Fold over siNT)
\end{tabular}

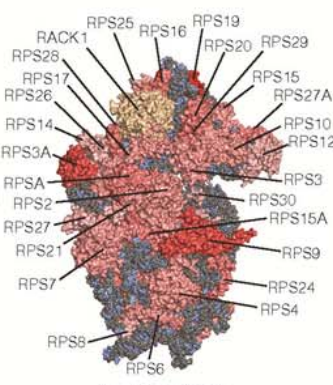

40S SOLVENT

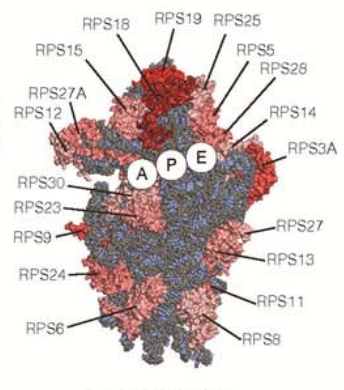

40S INTERFACE

\section{g}

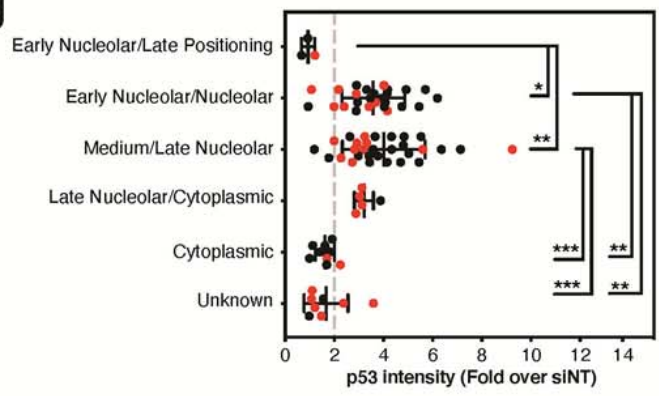


511 p53 homeostasis. Given the enrichment of ribosomal protein (RP) genes in our primary screen 512 dataset, we further investigated this group; depicted is the breakdown of screened RPs which were 513 p53 'positive' - 2-fold or greater increase in p53, and the proportion of which are located in the 514 large (60S) or small (40S) ribosome subunit, shown in (a). We further verified the p53 result of 515 approximately $50 \%$ of the RP genes (when depleted using siRNAs for 72 hours) with quantitative 516 p53 analysis (Alphascreen) in A549 cells (note genes associated with DBA are highlighted in red) 517 (b). We selected candidates which were 'p53 positive' (RPS18, RPL21, RPS19) and 'p53 negative' 518 (RPL5, RPL11, RPL22, RPL28) to confirm knockdown at the protein level, and determined p53 519 and p21 protein levels using western blot analysis in A549 cells (c, representative blot of $n=3$ 520 experiments). Cells depleted of each RP were then subjected to ribosome subunit analysis (performed under high salt conditions, d), to determine the effect of depletion on $60 \mathrm{~S}$ and $40 \mathrm{~S}$ 522 subunits, as well as polysome analysis (e). We rescreened the RP genes (to incorporate those which 523 were not assayed in the primary screen into the dataset), and mapped the p53 intensity of each RP 524 from the screen onto the near-atomic structure of the human ribosomal $60 \mathrm{~S}$ and $40 \mathrm{~S}$ subunits 525 resolved by Khatter and colleagues (PDB ID: 4UG0) ${ }^{13}$ (f), to determine if there were any patterns or 526 regions of the ribosome where $\mathrm{p} 53$ intensity was focused $(\mathrm{TE}=$ tunnel exit, $\mathrm{A}=$ 527 acceptor/aminoacyl-tRNA site, $\mathrm{P}=$ peptidyl-tRNA site, $\mathrm{E}=$ exit site). Comparison of the timing of 528 RP incorporation into the ribosome subunit (as tabulated by de la Cruz and colleagues ${ }^{14}$ ) with p53 529 intensity when the RP was depleted using siRNA (g). Data presented as mean -/+ SD, statistical 530 analysis: one-way ANOVA with Tukey's multiple comparison test, ${ }^{* * *} p<0.001,{ }^{* *} p<0.01,{ }^{*} p<$ 5310.05 ; red circles indicate 40S subunit RPs, black circles indicate 60S subunit RPs. Alphascreen 532 analysis performed $n=3-5$ biological experiments; ribosome subunit/polysome profiles, minimum $533 \mathrm{n}=3$ biological experiments per candidate. 


\section{Hannan et al Fig. 3}
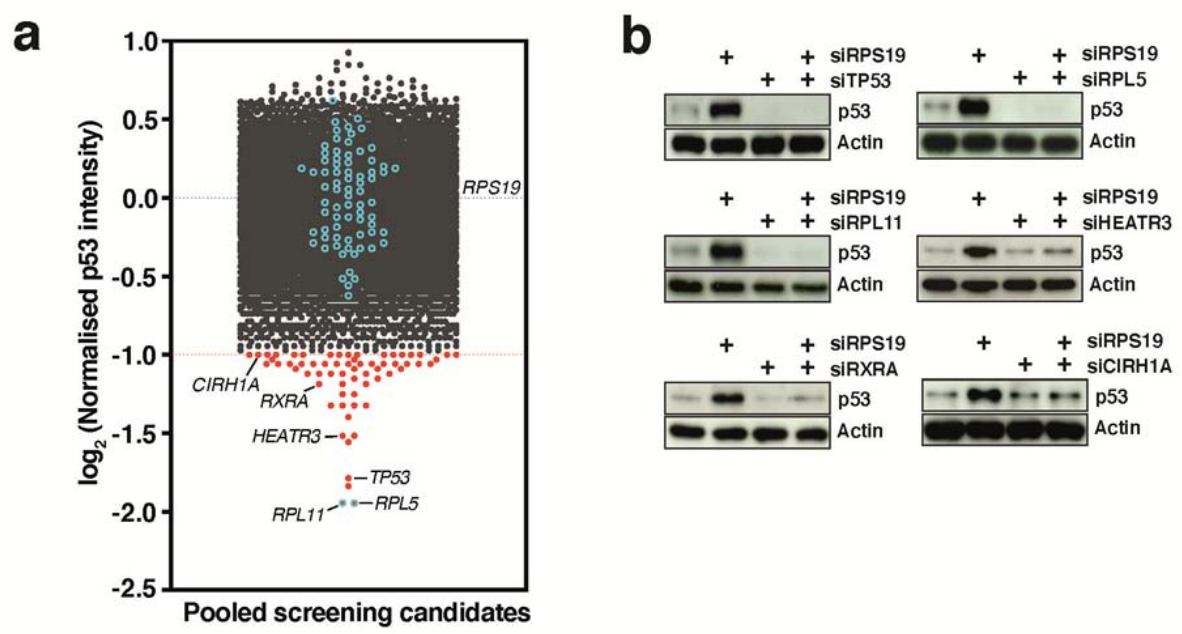

535 Figure 3: High-throughput screening for modifiers of ribosomal stress due to activation of the

536 canonical nucleolar surveillance pathway (NSP). In a similar approach (outlined in Fig. 1A), we

537 performed a genome-wide RNAi screen to identify modifiers of ribosomal stress, by co-depleting

538 RPS19 with every gene in the genome. After conducting the screen, candidates were further triaged

539 using gene-expression data from RNAseq analysis of A549 cells depleted of RPS19 (RPKM cutoff

540 of 0.05 or greater) to yield 14,577 'expressed' screen candidates. The 'expressed' screen candidates

541 were then graphed normalised to RPS19 depletion (a); candidates in red are those with a $\log _{2}$ value

542 of $\leq-1$ (total 64 candidates). Ribosomal protein (RP) genes in the screening data are demarcated

543 with blue circles. A selection of these candidates (TP53, RPL5, RPL11, HEATR3, RXRA and

544 CIRH1A) were then further subjected to candidate-based validation in A549 cells (b); by co-

545 depletion of candidates with siRPS19 for 72 hours and analysed by western blotting (representative

546 of $n=3$ biological experiments). 
Hannan et al Fig. 4

a

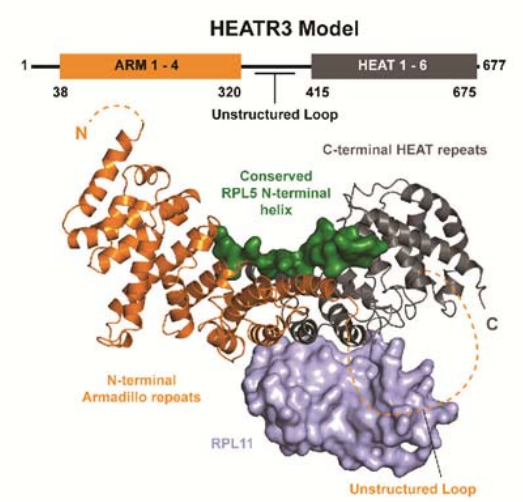

\section{b}

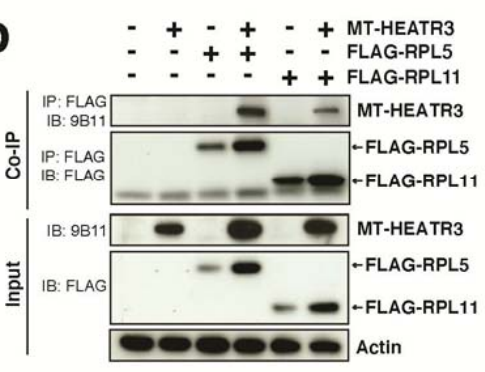

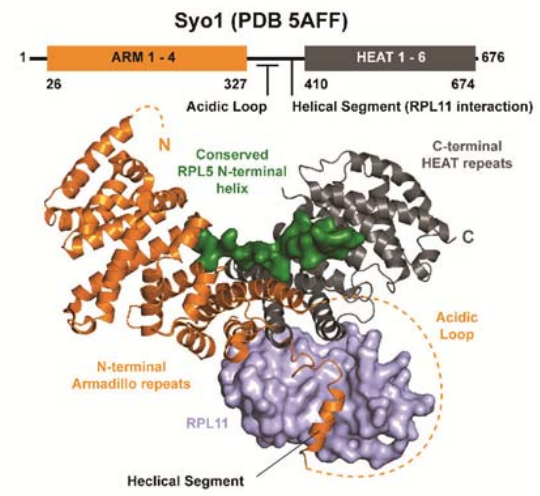
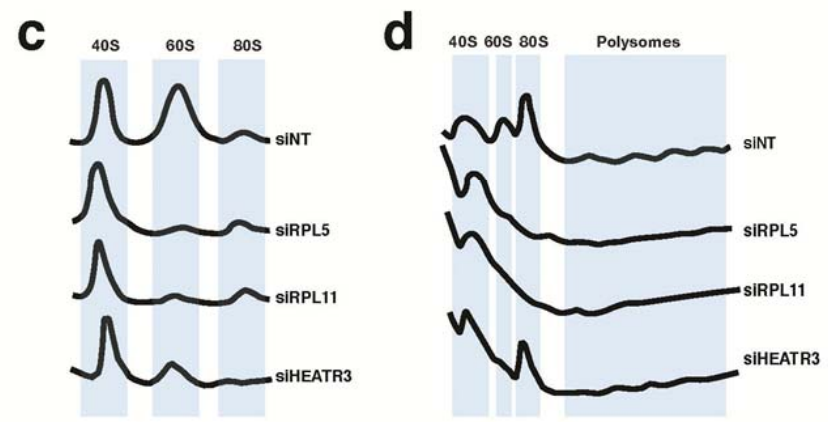
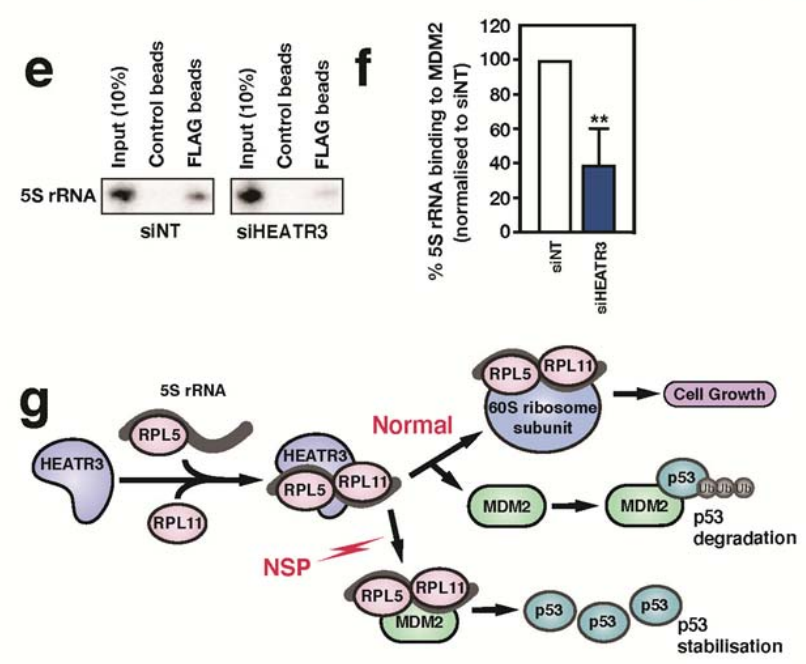

551 Figure 4: Analysis of the HEAT-repeat containing 3 (HEATR3) protein and its role in

552 ribosome and 5S-RNP biogenesis. Comparison between C. thermophilium Syo1 (ctSyo1) (PDB

$5535 \mathrm{AFF})^{36}$ and the predicted human HEATR3 structure (a). HEATR3 secondary structure and domain 554 modelling indicates the presence of an N-terminal Armadillo (ARM, orange), and a C-terminal 
555 HEAT repeat domain (dark grey), similar to the yeast Symportin 1 (Syo1) protein. A domain

556 schematic (to scale; top) and a cartoon model (bottom) are shown for each protein. Similar to the

557 ctSyo1 protein, HEATR3 contains four N-terminal Armadillo (ARM) repeats and six C-terminal

558 HEAT repeats. In the case for HEATR3, these regions are connected by a central, long and

559 unstructured loop, whereas ctSyo1 has an acidic loop with a helical segment (Glu389 to Gly399)

560 likely responsible, at least in part, for the binding of rpL11 (light blue, surface representation) to the

$561 \operatorname{protein}^{35}$. A conserved N-terminal segment of RPL5 (green, surface representation) may also

562 interact with HEATR3 (similar to Syo1). Co-immunoprecipitation (CoIP) analysis of human myc-

563 tagged HEATR3 (MT-HEATR3) with FLAG-tagged human RPL5 and RPL11 proteins in HEK293

564 cells (b). Ribosome (c) and polysome (d) profiling analysis of A549 cells depleted of RPL5, RPL11

565 or HEATR3 (and non-targeting siRNA, siNT) for 72 hours (note that the NT, RPL5 and RPL11

566 data traces presented here are already presented in Fig $2 \mathrm{~d}$ and e, and are replicated in this figure to

567 directly compare the effect of HEATR3 depletion with these conditions). Northern blot analysis of

568 the association of MDM2 with 5S rRNA after 48-hour HEATR3 depletion in U2OS cells

569 expressing FLAG-MDM2 (e) and quantitation (f). Schematic of the predicted role of HEATR3 in

570 5S-RNP biogenesis ("Normal") and the NSP (g). Error bars represent SD and statistical analysis

571 performed using unpaired student t-test ( $* * p<0.01, \mathrm{n}=3$ experiments).

572 


\section{Hannan et al Fig. 5}

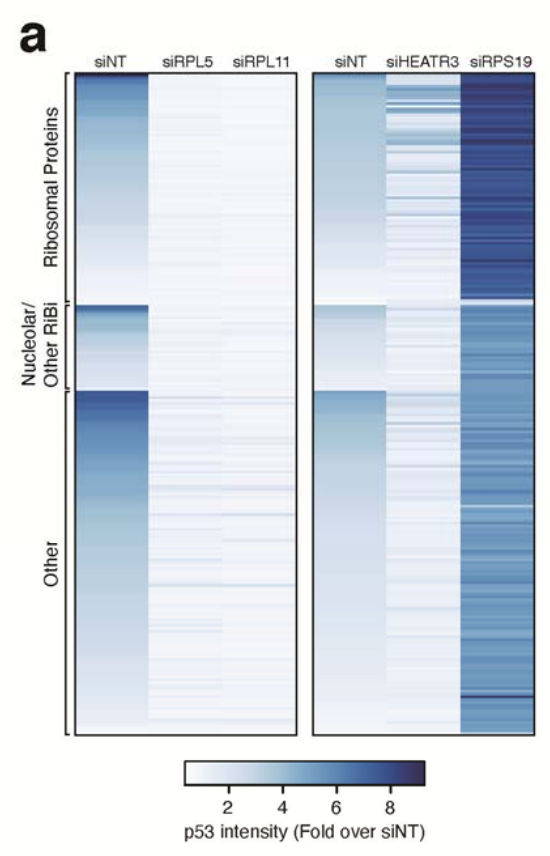

C
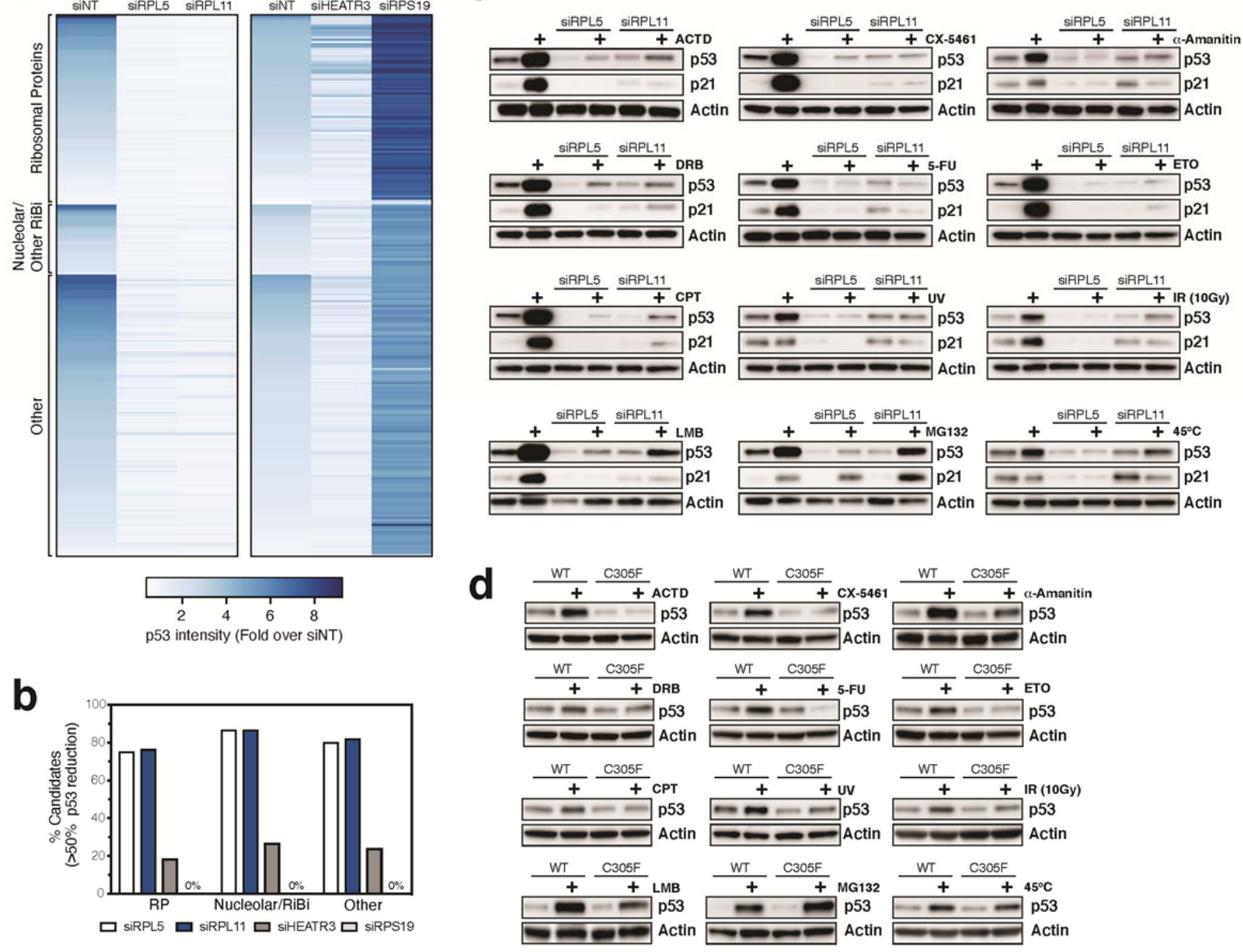

575 Figure 5: The NSP, via RPL5 and RPL11, is required to stabilise p53 in response to broad

576 range of genetic, pharmacological and pathophysiologic stresses. To identify which candidate

577 genes are required for the canonical NSP, we rescreened a selection of candidates from the primary

578 p53 stabilisation screen described in Fig. 3 (ribosomal proteins, nucleolar/RiBi candidates and

579 “other" p53 positive candidates, 232 genes in total) in the presence of non-targeting, RPL5, RPL11, 580 HEATR3 or RPS19 siRNAs in A549 cells (a). From this analysis, we further quantified the number 581 of candidates screened from each group (ribosomal proteins, nucleolar/RiBi and other) for which 582 their p53 response could be suppressed by $\geq 50 \%$ when co-depleted with RPL5, RPL11, HEATR3 583 or RPS19 siRNAs (b). We further tested a panel of pharmacological agents and pathophysiological 
584 stressors when A549 cells were depleted of RPL5 and RPL11 for 48 hours. Cells were treated for

58524 hours with pharmacological agents Actinomycin D (ACTD, $5 \mathrm{nM}), \alpha$-Amanitin $(2.5 \mu \mathrm{M})$,

586 Doxorubicin (DRB, $500 \mathrm{nM}), 5$-Fluoruracil (5-FU, $50 \mu \mathrm{M})$, Etoposide (ETO, $50 \mu \mathrm{M})$,

587 Camptothecin (CPT, $50 \mathrm{nM})$, Leptomycin B (LMB, $10 \mathrm{ng} / \mathrm{mL})$ or MG132 (10 $\mu \mathrm{M})$. Alternatively,

588 cells were treated with pathophysiological stressors UV $\left(50 \mathrm{~J} / \mathrm{m}^{2}\right)$, gamma irradiation (10Gy), or

589 subjected to heat shock $\left(45^{\circ} \mathrm{C}, 30\right.$ minutes $)$, then incubated at $37^{\circ} \mathrm{C}$ for 3 hours post treatment. At

590 the end of treatment, protein was harvested and subjected to western blot analysis for p53 and p21

591 protein expression (c). The same panel of stressors (and treatment conditions) were testing on

592 mouse embryonic fibroblasts (MEFs) isolated from either Mdm2 wild-type (WT) or mice

593 homozygous for the Mdm2 C305F mutation (C305F) to determine p53 expression (d). $\mathrm{n}=3-4$

594 biological replicates for each condition, the most representative experiment for each treatment is 595 presented.

596

597

598 